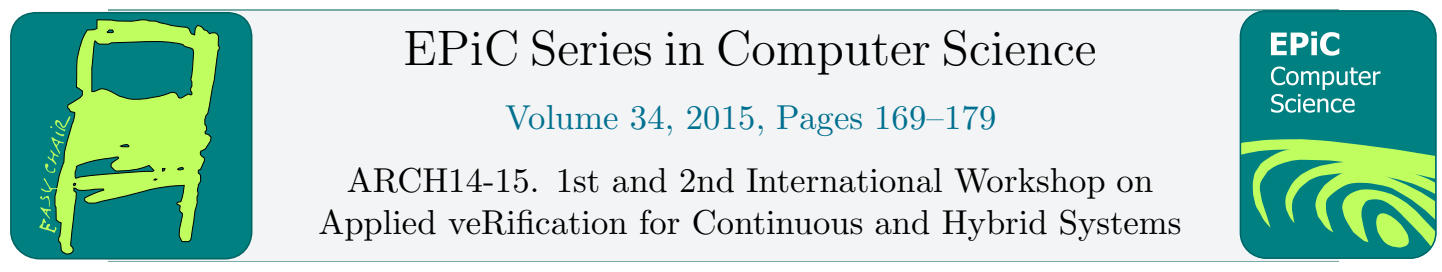

\title{
Optimizing Safe Control of a Networked Platoon of Trucks Using Reachability
}

\author{
Ibtissem Ben Makhlouf and Stefan Kowalewski \\ Informatik 11 - Embedded Software RWTH Aachen University \\ Ahornstrasse 55, 52074 Aachen, Germany \\ makhlouf, kowalewski@embedded.rwth-aachen.de
}

\begin{abstract}
The problem of conceiving a controller for networked systems is a challenging task because of the complex interaction of its different components with each other and also with the environment around them. The design process becomes more difficult if large-scaled systems are involved. We propose reachability analysis of continuous systems to guarantee control requirements which because of the complexity of the problem could not be taken into account during the control design. As example we suggest a large-scalable platoon of trucks. We use our own support function implementation to assess the performances of the obtained controlled platoon and then decide about the best performing controller.
\end{abstract}

\section{Introduction}

Networked control systems are multi-agent systems which exchange information via a communication network. This information is captured by on-board sensors and then sent to the other participants. This distributed and scalable structure presents a major challenge for the control design. The example of a networked platoon of trucks requires not only common control requirements like stability, overshoot limitation and fast responses, but also string stability requirement and collision-free guidance. Formulating all these requirements in the same framework leads to a complicated optimization problem. Furthermore, the complexity increases drastically with the dimension of the system. Many approaches have been proposed to overcome this problem. Many of them like those proposed in [8] and [5] ignore the control part involving the $H_{\infty}$ formulation related to string stability and solve the remaining part using the well scalable linear quadratic regulator (LQR)-control method. Other works solve only the $H_{\infty}$ optimization part [3]. Simulations are then used as tried-and-tested methods to test the ignored requirements. In [7] the problem is expressed as a mixed $H_{2} / H_{\infty}$ problem, which is then formulated as a linear matrix inequality (LMI) constraints by excluding the string stability condition. A controller for a platoon of 3 trucks has been computed. In [2], this example has been proposed as a benchmark for testing verification tools. Thereby the safety analysis under dropout of communication between the trucks has been investigated using reachability techniques. However the attempt to compute a controller for a scalable length of the platoon appears to be hard because of the 
intractability of the LMI-formulation.

Our strategy consists of using the same platoon model proposed in [7], which differs from the model proposed in [8] and [5], to perform a computation scheme for an LQR-based controller that is easy scalable to the number of trucks. The LQR-control design is then followed by a reachability analysis of the controlled platoon to check for the fulfillment of control performances not considered in the control design formulation for the sake of feasibility.

The rest of the paper is organized as follows. In Section 2, we handle the control design problem and describe the adopted control strategy. We introduce in Section 3 the reachability technique based on support functions and represent a numerical algorithm for computing reachable sets of linear continuous systems. We then illustrate in Section 4 our approach on a controlled platoon of 5 and 10 trucks and show the obtained reachable sets for the 5 trucks platoon as example followed by some comments. Finally, we wrap up this work with some concluding remarks in Section 5 .

\section{Control Design}

We first describe the infrastructure of a platoon of $N$ trucks and then explain the control strategy used to design the corresponding controller.

\subsection{Platoon Structure}

The platoon infrastructure is illustrated in Figure 1.

A platoon consists of $N$ trucks automatically controlled to follow a manually driven leader

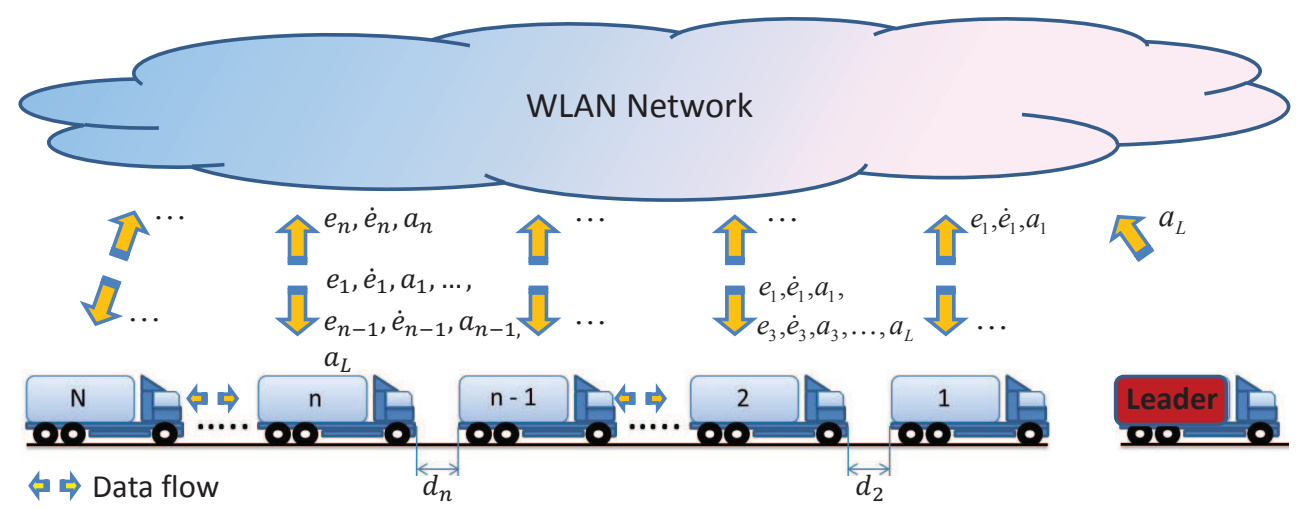

Figure 1: WLAN cooperative platoon with $\mathrm{N}$ trucks and a leader ahead.

ahead. Each truck $i$ is equipped with on-board sensors to measure the own relative distance $d_{i}$ to the truck in front, the relative velocity $\dot{e}_{i}$ and the acceleration $a_{i}$. It then computes the spacing error $e_{i}$ defined as the difference between $d_{i}$ and a reference distance $d_{r e f, i}$. The collected information $e_{i}, \dot{e}_{i}$ and $a_{i}$ is therefore sent by truck $i$ via a WLAN network to the other participants and receives in return via WLAN the data flow $e_{j}, \dot{e}_{j}$ and $a_{j}$ with $j \neq i$ from all other trucks inside the platoon.

Figure. 2 shows the experimental scaled autonomous trucks in a platoon formation at the Embedded Software Laboratory of RWTH Aachen. 


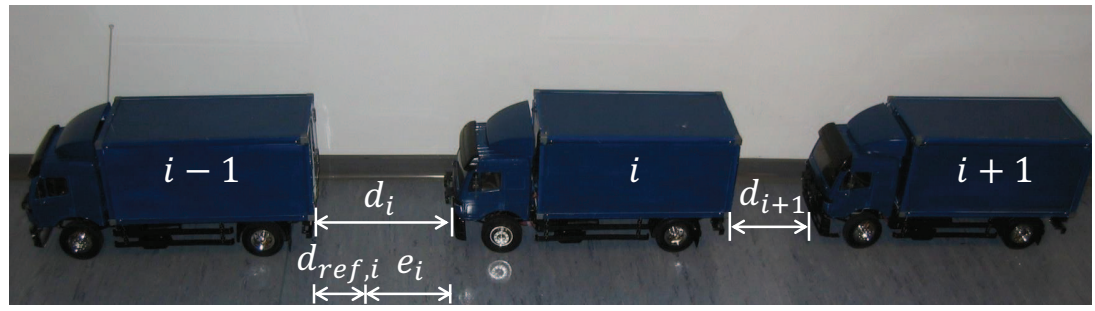

Figure 2: Experimental trucks and definition of the spacing error between successive trucks.

\section{2 $\quad$ Platoon Model}

The platoon is modeled as concentrated masses. The effective acceleration of each truck is governed by the drivetrain dynamics approximated by a linear first order filter [7]. Its dynamics is described by the following differential equations

$$
\begin{aligned}
& \ddot{e}_{i}=a_{i-1}-a_{i}, \\
& \dot{a}_{i}=-\frac{1}{T_{i}} a_{i}+\frac{1}{T_{i}} u_{i},
\end{aligned}
$$

with $T_{i}$ the time constant of the drivetrain of vehicle $i$ and $u_{i}$ the corresponding control signal.

\subsection{Control and Safety Requirements}

For the control design, we first have to stabilize the system while assuring a good disturbance rejection in terms of small and safe spacing errors $e_{i}$ with a reasonable control effort. To express mathematically further control objectives, we have to define different transfer functions and mappings. In [7], the following transfer functions and mapping have been defined

$$
\begin{aligned}
& G_{i}(s)=\frac{e_{i}(s)}{e_{i-1}(s)}, \\
& F_{v, i}(s)=\frac{\left(v_{i}-v_{L}\right)(s)}{a_{L}(s)}, F_{a, i}(s)=\frac{a_{i}(s)}{a_{L}(s)}, \\
& H: a_{L} \mapsto\left(e_{1}, \ldots, e_{n}, u_{1}, \ldots, u_{n}\right)^{T},
\end{aligned}
$$

with $H$ the mapping of $a_{L}$ to errors and control effort. The condition $\left\|G_{i}\right\|_{\infty}<1$ guarantees for string stability expressed formally by $e_{i} \leqslant e_{j}$ for $i<j$, where $<$ corresponds to the order of the trucks in the platoon. The conditions $\left\|F_{v, i}(s)\right\|_{\infty} \leqslant \gamma_{v}$ and $\left\|F_{v, i}(s)\right\|_{\infty} \leqslant \gamma_{a}$ maintain, however, the velocities and accelerations for all the followers respectively below $\gamma_{v}\left\|a_{L}\right\|_{\infty}$ and $\gamma_{a}\left\|a_{L}\right\|_{\infty}$, thereby limiting the upper bounds of the overshoots on velocity and acceleration with respect to the acceleration of the leader $a_{L}$ considered here as a disturbance. The control problem is formulated in [7] as a mixed $H_{2} / H_{\infty}$ optimization problem:

$$
\begin{aligned}
& \min \alpha .\|F\|_{\infty}+\beta .\|H\|_{2} \text { s.t. } \\
& \left\|G_{i}\right\|_{\infty}<1 \quad \forall i \\
& \left\|F_{., i}\right\|_{\infty}<\gamma . \forall i .
\end{aligned}
$$

where $F$ is either $F_{v, i}(s), F_{a, i}(s)$ or a combination of both. The $\|\cdot\|_{2}$-norm minimization of $H$ guarantees hence for short distance errors with a low control effort. The minimization problem (3) results in a feedback matrix $K$. The control input is then given by $u=K x$, where 
$x=\left[\cdots e_{i}, \dot{e}_{i}, a_{i} \cdots\right]^{T} \in \mathbb{R}^{3 n}$ is the state vector and $u$ is the control vector of the whole platoon. The closed loop system can therefore be described by the following differential equation

$$
\dot{x}=A_{c l} x+B_{c l} a_{L},
$$

where $A_{c l}$ is a constant system matrix, $B_{c l}$ is a constant input matrix and $a_{L}$ the acceleration of the leader considered here as an uncertain input [1].

\subsection{Controller Computation}

Solving the problem (3) for a platoon of $N$ trucks is challenging. In [7], a solution based on LMI-formulation has been proposed for a platoon of 3 trucks without considering the string stability condition and the overshoot limitation conditions. An extension of the LMI-optimization problem for more than 3 trucks has been revealed to be difficult, after discussions with the authors of [7]. For this reason, we decided to consider just the $H_{2}$ part of problem 3 and verify the other conditions with reachability technique. We use LQR-design [9] to solve this control problem and consequently compute a feedback matrix $K$ for the whole platoon. We illustrate our strategy with a platoon of 5,10 and 15 trucks. We take a time constant of the drivetrain equal to $T_{i}=0.5 \mathrm{~s}$ for all platoon members. Let $\gamma=1 / T_{i}$, the platoon dynamics is described by the following equation

$$
\dot{x}=A \cdot x+B_{2} \cdot u+B_{1} \cdot a_{L} .
$$

given in detail by (9).

The control task consists in computing a feedback matrix $K$ such $u=K . x$ by minimizing the following cost function

$$
J(u)=\int_{0}^{\infty}\left[x^{T} Q x+u^{T} R u\right] d t
$$

where $Q \geqslant 0$ and $R>0$ are adequately chosen matrices. The LQR-controller then takes the following form

$$
u=-R^{-1} B_{2}^{T} P x
$$

The matrix $P$ is a positive semidefinite solution of the Riccati equation

$$
P A+A^{T} P-Q+P B R^{-1} B^{T} P=0 .
$$



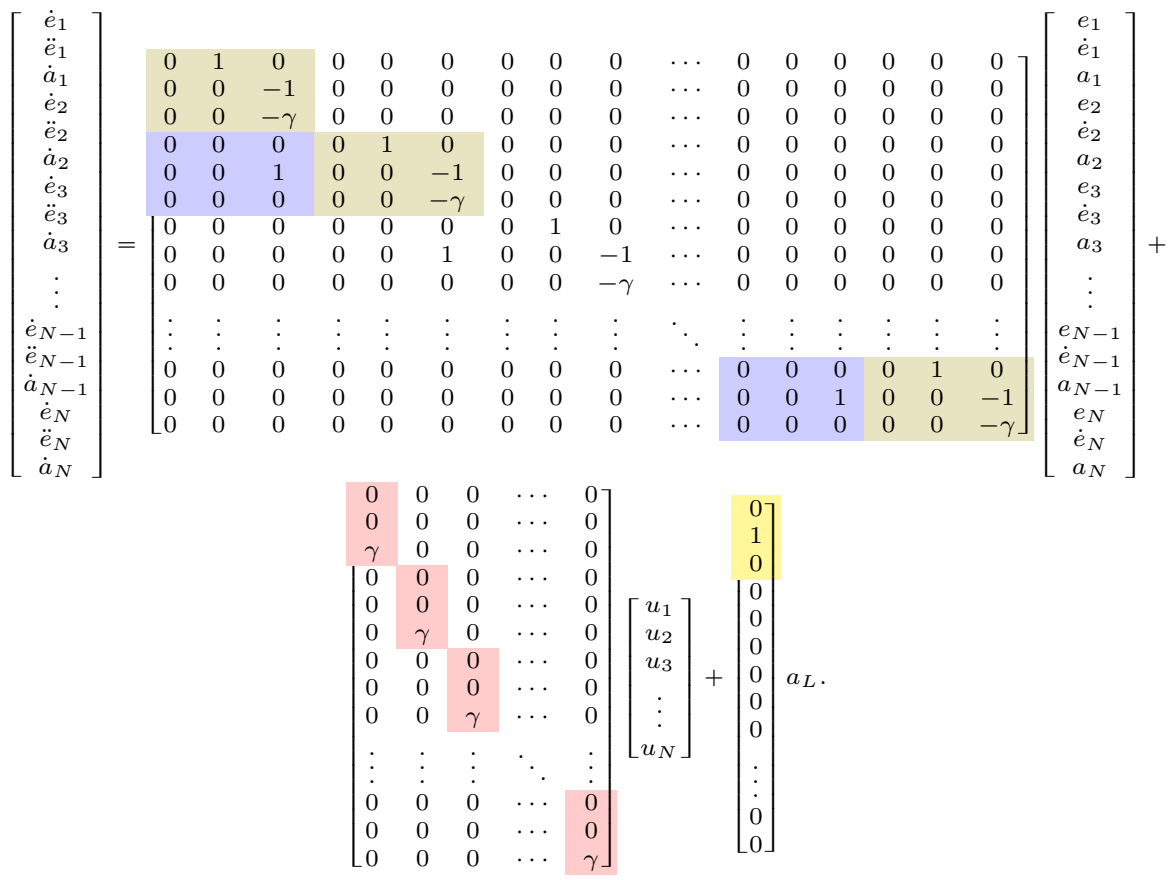

A reasonably simple choice for the weighting matrices $Q$ and $R$ involved in the minimization of the LQR-criteria (6) has led to the results of Figures 3, 4 and 5.
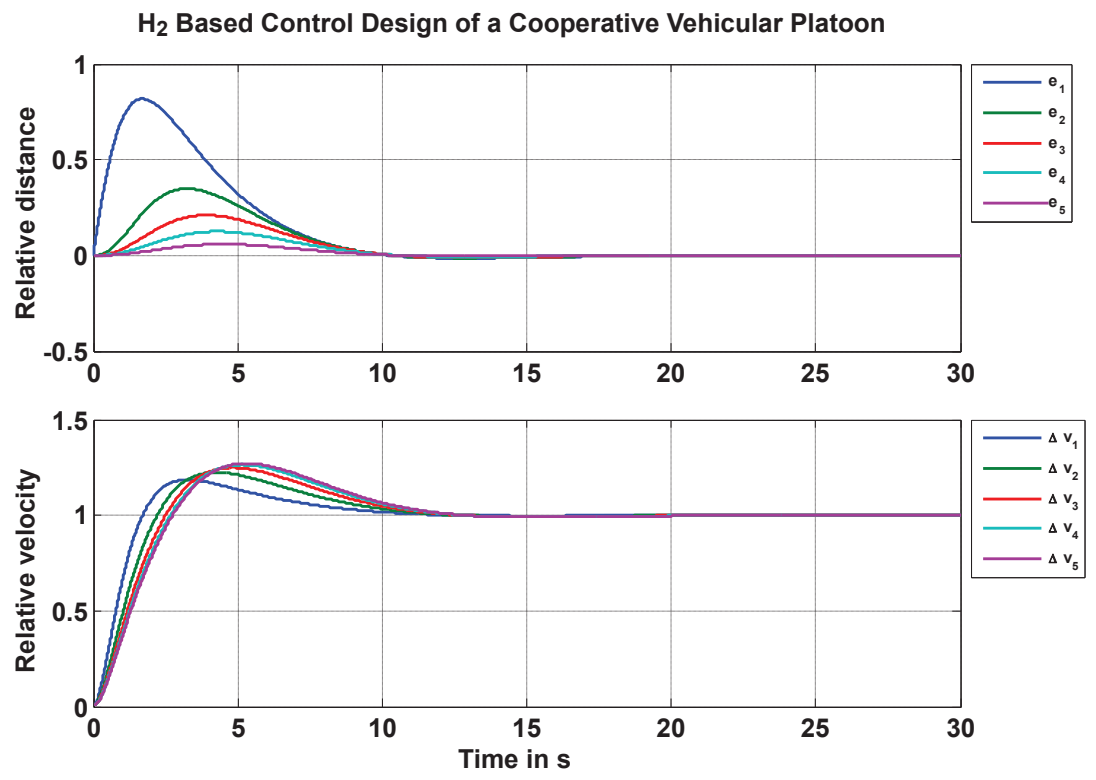

Figure 3: The responses of a controlled platoon of 5 trucks to a leader velocity in form of a $1 \mathrm{~m} / \mathrm{s}$ step.

We note that although only the problem of a fast decline of the errors with a minimum control 

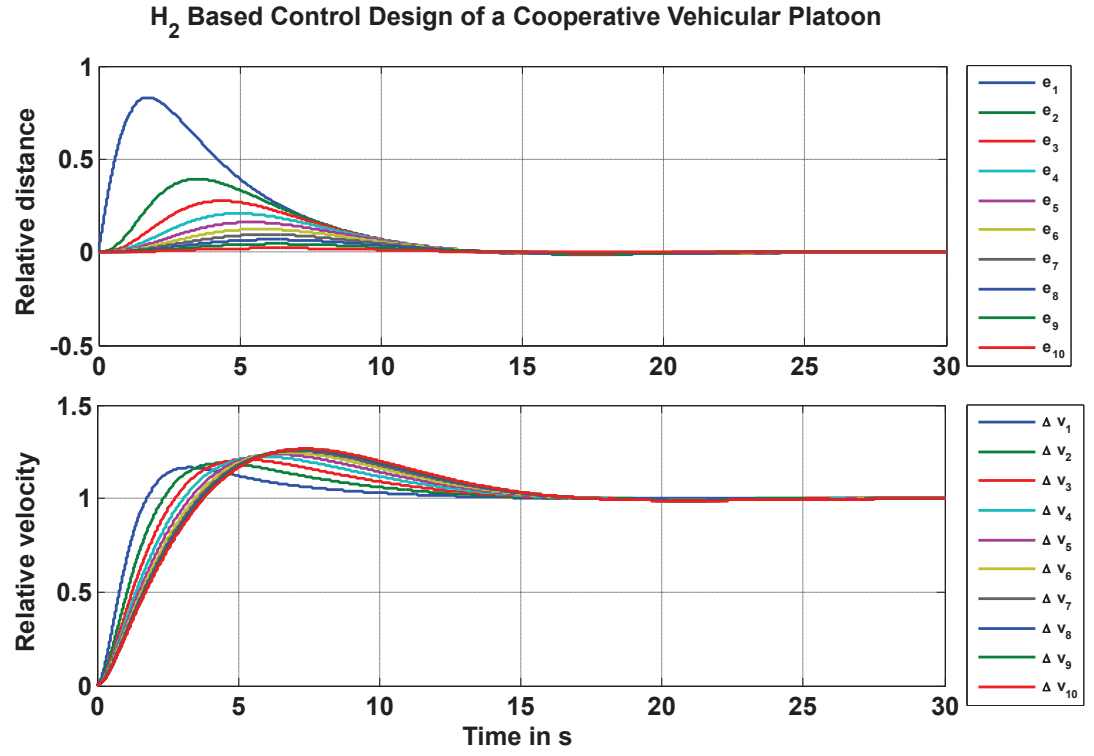

Figure 4: The responses of a controlled platoon of 10 trucks to a leader velocity in form of a $1 \mathrm{~m} / \mathrm{s}$ step.
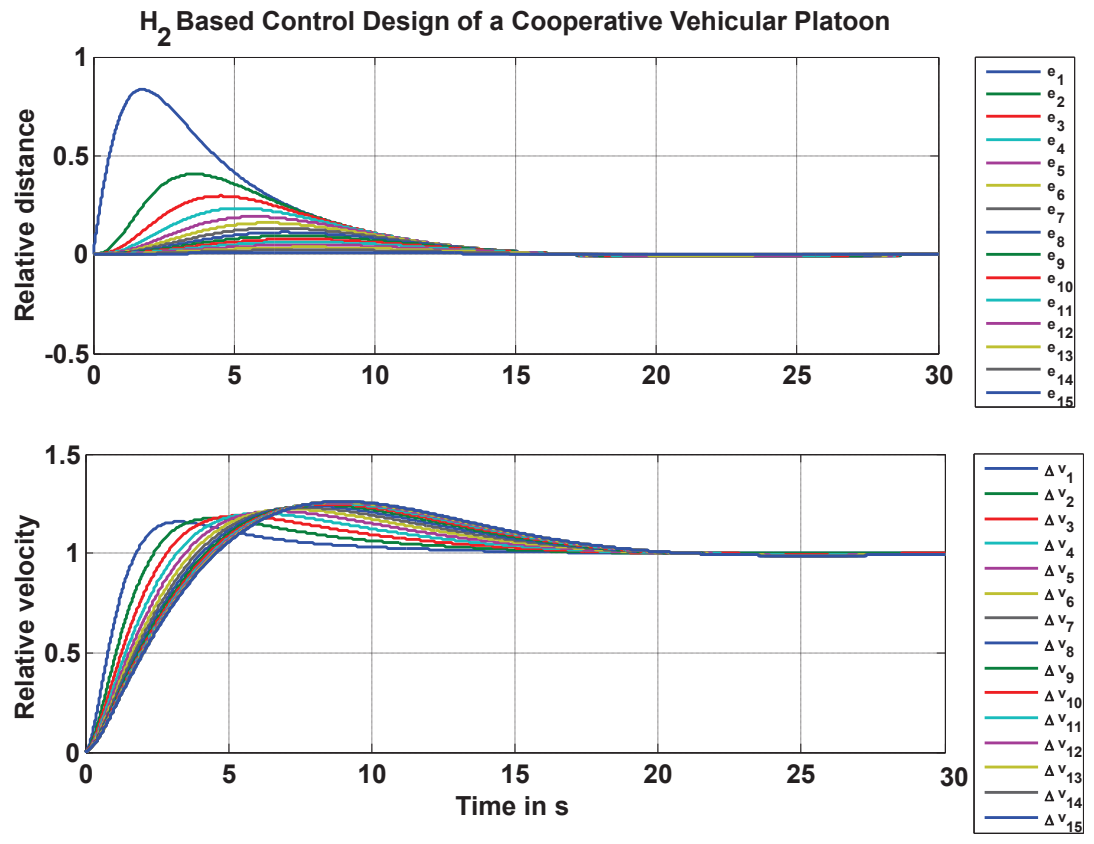

Figure 5: The responses of a controlled platoon of 15 trucks to a leader velocity in form of a $1 \mathrm{~m} / \mathrm{s}$ step. 
effort has been tackled by solving just the $H_{2}$ part of problem (3), an adequate choice of control parameters could limit the overshoots in the velocity and acceleration with an acceptable system time response. To guarantee for these performance criteria under all possible disturbances, we perform a reachability analysis in a next step.

\section{Reachability using Support functions}

We use reachability technique to first check if the response of system remains in acceptable ranges under a disturbance $a_{L}$ varying in $[-9,1] \mathrm{ms}^{-2}$. We will second verify if the string stability condition usually described by the attenuation of gaps along the platoon is kept. However, our main goal is to determine lower bounds for $d_{r e f, i}$ assuring collision-free driving. We opt for this study for a reachability technique based on support functions.

\subsection{Support functions}

A support function is an algebraic representation of geometric sets. Let $S \subset \mathbb{R}^{n}$ and $l \in \mathbb{R}^{n}$, the support function of $S$ is the function

$$
\begin{aligned}
\rho_{S}: & \mathbb{R}^{n} \longrightarrow \mathbb{R} \cup\{ \pm \infty\} \\
& l \longmapsto \rho_{S}(l):=\sup _{x \in S}\langle l, x\rangle
\end{aligned}
$$

where $\langle l, x\rangle$ is the dot product.

The following properties of the support function simplify their manipulation.

Let $S, S_{1}, S_{2} \subset \mathbb{R}^{n}$ be nonempty sets, $l \in \mathbb{R}^{n}, A \in \mathbb{R}^{n \times m}$ and $\lambda \geqslant 0$,

1. $\rho_{\lambda S}()=.\lambda \rho_{S}($.

2. $\rho_{S}(\lambda l)=\lambda \rho_{S}(l)$

3. $\rho_{A S}(l)=\rho_{S}\left(A^{T} l\right)$

4. $\rho_{S_{1} \oplus S_{2}}()=.\rho_{S_{1}}()+.\rho_{S_{2}}($.$) .$

These properties will be used in the next section to derive support function based recursive scheme for computing consecutive reachable sets.

\subsection{Reachability Algorithm}

Because of the fact that the controlled platoon dynamics take the form given by equation (4), we recall the recursion scheme given in [6] for the computation of reachable sets. This scheme is derived from the differential equation $\dot{x}=A x+B u$ with $A$ and $B$ constant matrices describing linear continuous systems. For a time step $r$ and a time horizon $T$, the reachable set $\Omega_{k}$ at time $t=k . r$ can be theoretically obtained by calling the set equation $\Omega_{k}=\Phi \Omega_{k-1} \oplus \mathcal{V}_{r}$ where $\Phi=e^{r A}$ and $\mathcal{V}_{r}$ is the input contribution for which an approximation will be proposed in the next paragraph. The input $u$ is supposed to be in the convex set $U \subset \mathbb{R}^{n}$. We use then support function properties to derive the following algebraic equations

$$
\begin{aligned}
\rho_{\Omega_{k}}(l) & =\rho_{\Phi \Omega_{k-1}}(l)+\rho_{\mathcal{V}_{r}}(l) \\
& =\rho_{\Omega_{k-1}}\left(\Phi^{T} l\right)+\rho_{\mathcal{V}_{r}}(l)
\end{aligned}
$$


It follows therefore

$$
\begin{aligned}
\rho_{\Omega_{k}}(l) & =\rho_{\Omega_{k-1}}\left(\Phi^{T} l\right)+\rho_{\mathcal{V}_{r}}(l) \\
& =\left[\rho_{\Omega_{k-2}}\left(\Phi^{T} \Phi^{T} l\right)+\rho_{\mathcal{V}_{r}}\left(\Phi^{T} l\right)\right]+\rho_{\mathcal{V}_{r}}(l) \\
& =\rho_{\Omega_{k-2}}\left(\Phi^{T^{2}} l\right)+\rho_{\mathcal{V}_{r}}\left(\Phi^{T} l\right)+\rho_{\mathcal{V}_{r}}(l) \\
& =\rho_{\Omega_{k-3}}\left(\Phi^{T^{3}} l\right)+\rho_{\mathcal{V}_{r}}\left(\Phi^{T^{2}} l\right)+\rho_{\mathcal{V}_{r}}\left(\Phi^{T} l\right)+\rho_{\mathcal{V}_{r}}(l) \\
& \vdots \\
& =\rho_{\Omega_{0}}\left(\Phi^{T^{k}} l\right)+\sum_{j=0}^{k-1} \rho_{\mathcal{V}_{r}}\left(\Phi^{T^{j}} l\right)
\end{aligned}
$$

If we adopt the notations $d_{k}=\Phi^{T^{k}} l$ and $s_{k}=\sum_{j=0}^{k-1} \rho_{\mathcal{V}_{r}}\left(\Phi^{T^{j}} l\right)$, the above equations can be implemented using Algorithm 1.

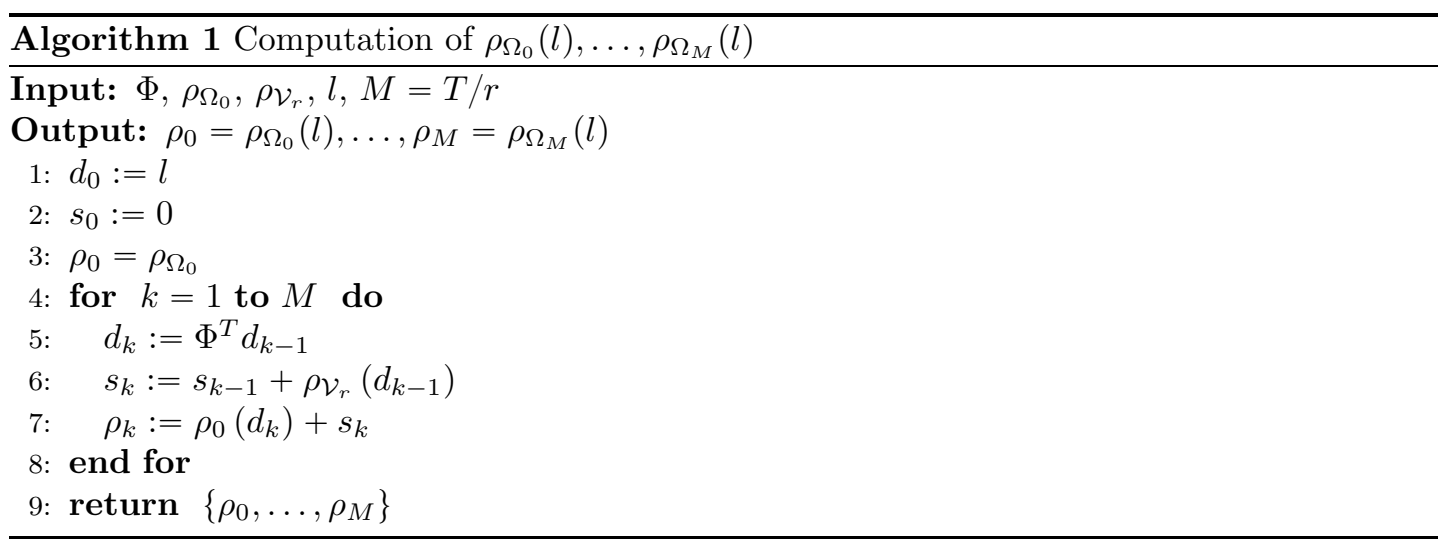

However, this algorithm requires for the initialization step the over-approximations for the input contribution $\rho_{\mathcal{V}_{r}}$ and the initial set $\rho_{\Omega_{0}}$. For the input contribution, we opt for the approximation

$$
\begin{aligned}
& \rho \mathcal{V}_{r}(l)=r \rho_{U}\left(B^{T} l\right)+\rho_{\mathcal{E}_{U}}(l), \text { with } \\
& \mathcal{E}_{U}=\square\left(|A|^{-2}\left(e^{r|A|}-I_{n}-r|A|\right)\right) \boxminus(A B U),
\end{aligned}
$$

proposed in [4] where $I_{n}$ is the identity matrix and $|A|$ is the component-wise absolute value operation of the matrix $A$. The operators $\square$ and $\square$ correspond respectively to the interval hull and the symmetric interval hull of sets.

We also use the method suggested in [4] to compute an approximation for the initial set. This is given as follows:

$$
\begin{aligned}
\rho_{\Omega_{0}}(l) & =\max _{\lambda \in[0,1]} \rho_{\Omega_{0, \lambda}}(l), \text { where } \\
\rho_{\Omega_{0, \lambda}}(l)= & (1-\lambda) \rho_{X_{0}}(l)+\lambda \rho_{e^{r A} X_{0}}(l)+\lambda r \rho_{B U}(l) \\
& +\lambda^{2} \rho_{\mathcal{E}_{U}}(l)+\rho_{\left(\lambda \mathcal{E}_{X_{0}}^{+} \cap(1-\lambda) \mathcal{E}_{X_{0}}^{-}\right)}(l) \\
& =(1-\lambda) \rho_{X_{0}}(l)+\lambda \rho_{X_{0}}\left(\left(e^{r A}\right)^{T} l\right)+\lambda r \rho_{U}\left(B^{T} l\right) \\
& +\lambda^{2} \rho_{\mathcal{E}_{U}}(l)+\rho_{\left(\lambda \mathcal{E}_{X_{0}}^{+} \cap(1-\lambda) \mathcal{E}_{X_{0}}^{-}\right)}(l), \text { and } \\
& =\bullet\left(|A|^{-2}\left(e^{r|A|}-I_{n}-r|A|\right) \boxminus\left(A^{2} X_{0}\right)\right) \\
\mathcal{E}_{X_{0}}^{+} & =\bullet\left(|A|^{-2}\left(e^{r|A|}-I_{n}-r|A|\right) \bullet\left(A^{2} e^{r A} X_{0}\right)\right) \\
\mathcal{E}_{X_{0}}^{-} & =
\end{aligned}
$$


For the computation of the part with the intersection, we use the following property of support functions. Let $S, S_{1}, S_{2} \subset \mathbb{R}^{n}$ be nonempty sets, $l \in \mathbb{R}^{n}$ then

$$
\rho_{S_{1} \cap S_{2}}(l) \leqslant \min \left(\rho_{S_{1}}(l), \rho_{S_{2}}(l)\right) .
$$

To get the polyhedron approximating the reachable sets, we call Algorithm 1 for each direction element of a predefined template of directions $D=\left\{l_{1}, \ldots, l_{m}\right\}$.

\section{Results}

As an example, we compute the reachable sets of a platoon of 5 trucks controlled using the LQRcontrol strategy described above. The state vector is equal to $x=\left(e_{1}, \dot{e_{2}}, a_{1}, \ldots, e_{5}, \dot{e_{5}}, a_{5}\right) \in \mathbb{R}^{15}$ and the input equal to $a_{L}$ is in $[-9,1] \mathrm{ms}^{-2}$. For the verification, we choose a time horizon $T=30 \mathrm{~s}$, a time step $r=0.01 \mathrm{~s}$ and octagonal directions. The choice of the time horizon is based on the results of the simulations of Figure 3. We note that the system reaches a stable state after $15 \mathrm{~s}$. This time increases with the number of trucks as shown in Figures 4 and 5 . We push this time limit to $30 s$ to ensure the maximum reachable set. The obtained results are shown in Figure 6.

\begin{tabular}{|c||c|c|c|c|c|}
\hline $\mathrm{i}$ & 1 & 2 & 3 & 4 & 5 \\
\hline \hline$d_{\text {ref }, i}$ & $35 \mathrm{~m}$ & $16 \mathrm{~m}$ & $10 \mathrm{~m}$ & $7 \mathrm{~m}$ & $3 \mathrm{~m}$ \\
\hline
\end{tabular}

Table 1: Minimum allowed safe distances.

The boundaries of the reachable sets in Figure 6 give the ranges of each state variables. For the velocity and the acceleration, we consider only the positive part. We note first of all that the maximum values of the velocity and the acceleration for each trucks, which correspond to maximum overshoots, remain under acceptable thresholds. The minimum values of different errors $e_{i}, i=1, \ldots, 5$, however, correspond to the minimum allowed safe distances between successive trucks.

These values are listed in Table 1 . We observe furthermore that the gaps attenuate along the platoon. The computed controller fulfills thereby all expected requirements.

\section{Conclusion}

The paper suggests an approach to reduce the problem of control design of networked systems to some requirements leading to an efficient scalable formulation of the control problem and to check after computing the appropriate controller if other ignored requirements are met. We propose a reachability analysis based on support functions to get an overview of the dynamical behavior of the state variables under disturbances. As case study, a scalable platoon is used. For a platoon of 5 trucks, we compute an LQR-based controller taking into account just a fast attenuation of errors with a minimum control effort. Requirements like limiting overshoots of velocity and acceleration under disturbances as well as string stability are checked using reachability. If we notice that the obtained results do not meet the control requirements, the controller will be consequently recomputed by choosing different weighting matrices $Q$ and $R$. 


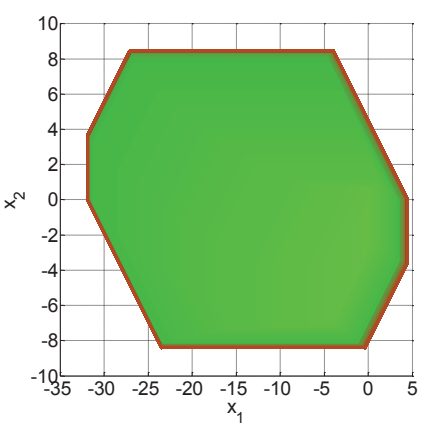

(a)

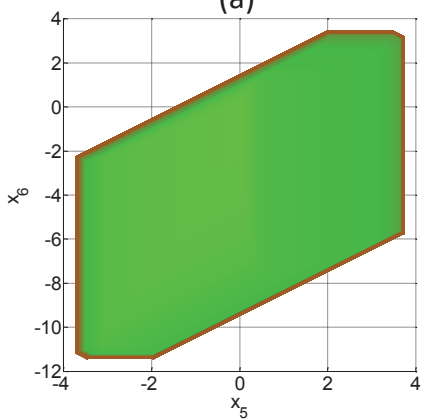

(c)

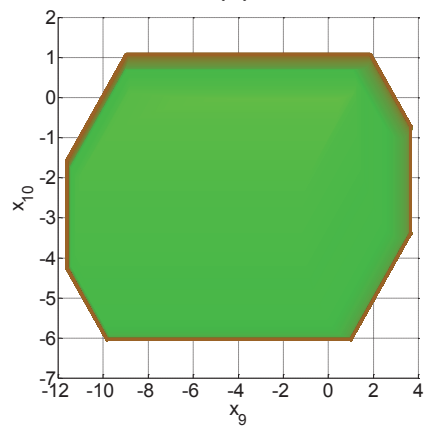

(e)

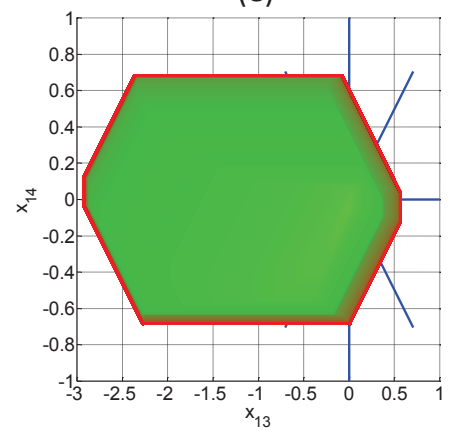

(g)

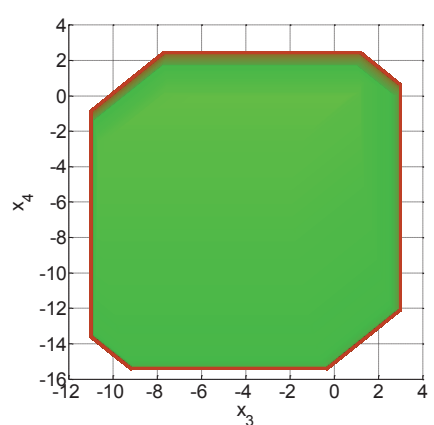

(b)

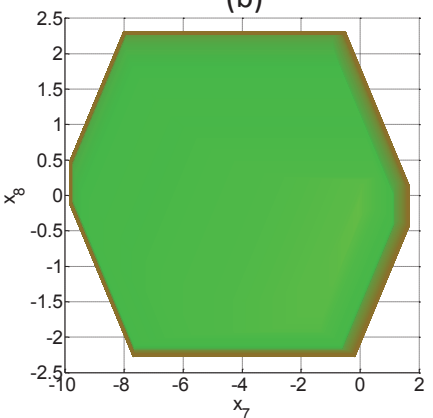

(d)

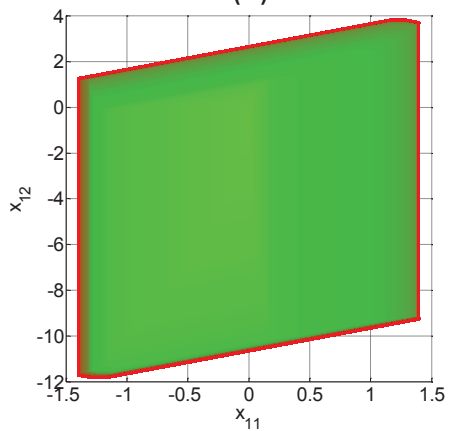

(f)

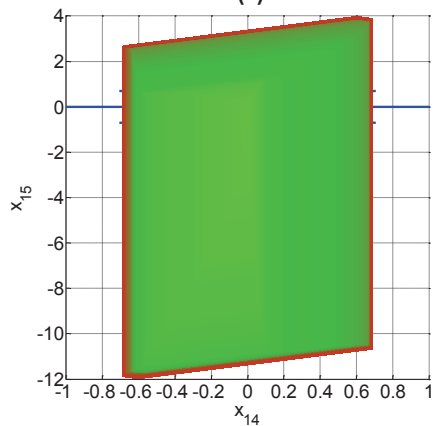

(h)

Figure 6: The reachable sets of the platoon of 5 trucks. 


\section{References}

[1] I. Ben Makhlouf, H. Diab, and S. Kowalewski. Safety verification of a controlled cooperative platoon under loss of communication using zonotopes. In Proc. of the 4 th IFAC Conference on Analysis and Design of Hybrid Systems (ADHS 12), pages 333-338, Eindhoven, NL, 2012.

[2] I. Ben Makhlouf and S. Kowalewski. Networked cooperative platoon of vehicles for testing methods and verification tools. In ARCH14 CPSWeek Berlin, 2014.

[3] F. Farokhi, H. Sandberg, and K.H. Johansson. Optimal $H_{\infty}$ control design under model information limitations and state measurement constraints. In Decision and Control $(C D C)$, 2013 IEEE 52nd Annual Conference on, pages 6218-6225, Dec 2013.

[4] G. Frehse, C. Le Guernic, A. Donzé, S. Cotton, R. Ray, O. Lebeltel, R. Ripado, A. Girard, T. Dang, and O. Maler. Spaceex: Scalable verification of hybrid systems. In Shaz Qadeer Ganesh Gopalakrishnan, editor, Proc. 23rd International Conference on Computer Aided Verification (CAV), LNCS. Springer, 2011.

[5] X. Huppe, J. de Lafontaine, M. Beauregard, and F. Michaud. Guidance and control of a platoon of vehicles adapted to changing environment conditions. In $S M C$, pages 3091-3096. IEEE, 2003.

[6] C. Le Guernic. Reachability Analysis of Hybrid Systems with Linear Continuous Dynamics. $\mathrm{PhD}$ thesis, VERIMAG, Oct. 2009.

[7] J. P. Maschuw, G. C. Keßler, and D. Abel. LMI-based control of vehicle platoons for robust longitudinal guidance. In IFAC World Congress 2008, Seoul, Jul. 2008.

[8] B. Shu and B. Bamieh. Robust $\mathrm{H}_{2}$ control of vehicular strings. ASME J. Dynamics, Measurement and Control, 1996.

[9] K. Zhou, J. C. Doyle, K. Glover, et al. Robust and optimal control, volume 40. Prentice hall New Jersey, 1996. 